\title{
Antisense EGFR sequence enhances apoptosis in a hu- man hepatoma cell line BEL-7404
}

FU TaO ${ }^{1 *}, \mathrm{He} \mathrm{LiU}^{*}, \mathrm{Fan} \mathrm{LIU}^{* *} \mathrm{JUN}$ GU***, Wan Li JIANG*, Yong HuA XU ${ }^{2 *}$

* Laboratory of Cellular and Molecular Oncology, Shanghai Institute of Cell Biology, Chinese Academy of Sciences, shanghai 200031

** Shanghai Bureau of Hygiene

*** Laboratory of Molecular Biology, Naval Medical Research Institute, Shanghai 200433, China

\begin{abstract}
Effects of antisense epidermal growth factor receptor (EGFR) sequence on apoptotic cell death were examined in a human hepatoma cell line BEL-7404 cells. In the cells of JX-1, a sub clone of BEL-7404 stably transfected with antisense EGFR vector (Cell Research, 3:75, 1993), an enhanced rate $(9.5 \%)$ of spontaneous apoptosis was detected by flow cytometry, whereas the rates of spontaneous apoptosis in JX-0 cells, a sub-clone of BEL-7404 transfected by control vector, and the parent BEL-7404 cells were almost equal and about $1.7 \%$. Serum-starvation for $72 \mathrm{~h}$ increased the rate of apoptosis of JX-1 cells up to $33.7 \%$, while JX-0 and BEL-7404 cells, under the same condition, produced less than $5 \%$ of apoptotic cells. Observation with electron microscope demonstrated that condensation and fragmentation of chromatin and formation of apoptotic bodies often occurred in JX-1 cells, especially during serumstarvation. These results, combined with the data of DNA fragmentation Elisa test, suggested that antisense EGFR sequence enhances apoptosis in the human hepatoma cells.
\end{abstract}

1. Present address: Hollings Cancer Center, Medical University of South Carolina, Chasleston, SC 29403, USA

2. To whom all correspondence should be addressed 
Antisense EGFR enhances apoptosis in hepatoma cells

Comparison of intracellular $\mathrm{Ca}^{2+}$ level and the responsiveness of JX-1 cells to the induced action of EGF and tharpsigargin (TG) treatment with that of control JX-0 cells indicated that antisense EGFR might interrupt the EGF/EGFR signaling pathway resulting in the decreass of intracellular $\mathrm{Ca}^{2+}$ pool content as well as the responsiveness of these cells to the extracellular signals. These findings suggest that antisense EGFR either directly or indirectly regulates $\mathrm{Ca}^{2+}$ storage in endoplasmic reticulum, thereby enhances apoptosis in the human hepatoma cells.

Key words: Antisense EGFR, hepatoma cells, apoptosis.

\section{INTRODUCTION}

Apoptosis is an active form of programmed cell death different from necrosis. Like cell proliferation and differentiation, apoptosis is a characteristic event or process which is regulated by both intracellular and extracellular signals[1-3]. Recently, the increasing evidence implies that alteration in apoptosis contribute to tumorogenesis and thus treatment designed to alter apoptosis may have the potential to change the progression of cancer[4].

The epidermal growth factor receptor (EGFR) is a $170 \mathrm{KD}$ glycoprotein which contains essentially an extracellular binding domain, a transmembrane domain and an intracellular protein kinase domain. The EGFR tyrosine kinase-mediated signal transduction pathways have been considered to regulate many cellular processes. Amplification and overexpression of the EGFR has been detected in a number of human tumors of epithelial origin[5]. The sequence homology has been found between intracelluar portion of the EGFR and $\mathrm{gp}^{68 \mathrm{ver}-\mathrm{er} B}$, the transforming protein of oncogene v-erbB [6]. The EGFR activation has been implicated in autocrine stimulation of cell growth in many experimental system[7]. Therefore, the EGFR might be responsible for the genesis and the maintenance of malignant properties of certain types of tumors and it appears to be a good target for antitumor therapy.

In the previous studies, we have demonstrated the EGFR gene expression and mitogenic effects of EGF in cells of a human hepatoma cell line BEL-7404[8]. Furthermore, we have obtained an antisense EGFR vector transfected BEL-7404 cell clone, termed JX-1 cells, showing amazing alteration in growth behaviors[9]. We describe here that antisense EGFR sequence enhances apoptotic cell death in the transfected JX-1 cells and its underlying mechanism will be discussed. 
Fu T et al.

\section{MATERIALS AND METHODS}

\section{Materials}

Fluo-3/AM, propidium iodide and thapsigargin were purchased from Sigma (St. Louis, MO, USA). EGF was the product from Gibco BRL (Gaithersburg, MD, USA).

\section{Cell culture}

Human hepatoma cell line BEL-7404 was established in our Institute. BEL-7404 cells and the derivatives were grown in Dulbocco's modified Eagles's medium (DMEM, Gibco, Grand Island, NY, USA) supplemented with $13 \%$ fetal calf serum, $100 \mathrm{u} / \mathrm{ml}$ penicillin and $100 \mathrm{mg} / \mathrm{ml}$ streptomycin in $5 \% \mathrm{CO}_{2}$ incubator at $37^{\circ} \mathrm{C}$ and cultured medium was changed every $48 \mathrm{~h}$.

\section{Stable transfectants}

The recombinant plasmid containing antisense EGFR sequence was co-transferred with pSV2neo into BEL-7404 cells by electroporation, followed by selection and cloning in the presence of G418 $(400 \mu \mathrm{g} / \mathrm{ml})$. The parent vector plasmid pEV-1 was used as control for transfection. Two representative cell lines JX-1 transfected with antisense EGFR sequence and JX-0 transfected with control vector pEV-1 were cloned for further analysis.

\section{Electron microscopy}

For transmission electron microscopy analysis, exponentially growing JX-1 cell in monolayer culture were collected by trypsinizing. The cells were fixed in $4 \%$ glutaraldehyde, postfixed in $1 \%$ $\mathrm{OsO}_{4}$, and embedded with Epon618. Ultrathin sections were cut and stained with uranyl acetate and lead citrate and viewed in a JEWl00B electron microscope.

\section{Flow cytometry}

The cells grown in monolayer culture were trypsinized and centrifuged at $250 \times \mathrm{g}$ for $5 \mathrm{~min}$. The cell pellet was resnspended in $1 \mathrm{ml}$ hypotonic fluorochrome solution (propidium iodide $50 \mu \mathrm{g} / \mathrm{ml}$ in $0.1 \%$ sodium citrate plus $0.1 \%$ Triton X-100, Sigma). The sample was kept at $4{ }^{\circ} \mathrm{C}$ in dark overnight. The propidium iodide fluorescence of individual cells was measured by using a FACStar Plus flow cytometry (Becton and Dickson). The cells with subdiploid DNA content were considered as apoptotic cells. Each value represented the mean of 10,000 cells.

\section{DNA fragmentation Elisa assay}

Cellular DNA fragmentation was measured by using a commercial available DNA fragmentation Elisa kit (Boehringer Mannheim) which is based on the quantitative sandwisch enzyme immunoassay principle using two mouse monoclonal antibodies directed against DNA and BrdU respectively. This allows the specific detection and quantification of Brdu-labeled DNA fragments. Briefly, the cells $\left(1 \times 10^{4}\right)$ were seeded to 96 -weU flat bottom cell culture plate (Corning). Exponentially growing cells were incubated with $10 \mu M$ BrdU for $12 \mathrm{~h}$ in $5 \% \mathrm{CO}_{2}$ incubator at $37^{\circ} \mathrm{C}$ and then lysed by adding lysis buffer. After lysis, the samples were centrifuged at $250 \times \mathrm{g}$ for $10 \mathrm{~min}$ and $100 \mu 1$ of supernatant removed from each sample for Elisa assay. The samples were added to microtiter plate precoated with anti-DNA antibody and incubated for $90 \mathrm{~min}$. After the samples were incubated with anti-BrdU peroxidase for another $90 \mathrm{~min}$, TMB substrate was added to plate. After incubation in the dark for $20 \mathrm{~min}$, the reaction was terminated by adding $25 \mu \mathrm{l}$ of $1 M \mathrm{H}_{2} \mathrm{SO}_{4}$ to each well. Measurement of absorbance at $450 \mathrm{~nm}$ was performed using microplate reader (Bio-Rad).

\section{Measurement of intracellular free $\mathrm{Ca}^{2+}$ concentration}

Cytosolic $\mathrm{Ca}^{2+}$ concentration was measured by fluorometry of furo-2-1oaded or fluo-3-1oaded cells as described[18]. 
Antisense EGFR enhances apoptosis in hepatoma cells

\section{RESULTS AND DISCUSSION}

EGF/EGFR pathway is known as an important regulating network for the growth control in either normal cells or malignant cells. We reported here the study of the role of EGFR in apoptosis of human hapatoma cells using antisense EGFR vector transfectants as a model. Flow cytometry combined with propidium iodide-staining showed that an increased number of sub-G1 phase cells, which contained subdiploid DNA, occurred in antisense EGFR transfected cell line JX-1 cells in comparison to those in control vector transfectants JX-0 cells and the parent BEL-7404 cells (Tab 1, left column). The percentage of cells with subdiploid DNA content in JX-1 cells was $9.5 \%$,

Tab 1. The effects of serum starvation on apoptosis of BEL-7404, JX-0 and JX-1 cells*

\begin{tabular}{ccc}
\hline & \% cells with subdiploid DNA content $(\mathrm{M} \pm$ S.D., $\mathrm{n}=3)$ \\
\cline { 2 - 3 } Cells lines & Serum $(+)$ & Serum $(-)$ \\
\hline Bel-7404 & $1.65 \pm 0.24$ & $2.28 \pm 0.43$ \\
JX-0 & $1.72 \pm 0.15$ & $3.25 \pm 0.38$ \\
JX-1 & $9.51 \pm 1.69$ & $18.41 \pm 1.03$ \\
\hline
\end{tabular}

$* 80 \%$ confluent cells of BEL-7404, JX-0 and JX-1 were cultured in the fresh DMEM with or without $13 \%$ FCS for $24 \mathrm{~h}$ before harvesting.

whereas those in JX-0 and BEL-7404 cells were similar and about 1.7\%, indicating an enhanced rate of spontaneous apoptotic cell death in JX-1 cells. In the previous study[9], we have demonstrated that BEL-7404 and control vector transfectants JX-0 cells can grow in serum-free medium, while JX-1 cells can not survive during the serum-starvation. In the present study, it was found that after serumstarvation for $24 \mathrm{~h}$, the rate of apoptosis of JX-1 cells was increased up to $18.4 \%$, but JX-0 and BEL-7404 cells only showed less than 2-3\% apoptotic cells (Tab 1, right column). When the serum-starvation was elongated to $72 \mathrm{~h}$, as shown in Fig 1 , the rate of apoptotic cells with subdiploid DNA content were increased up to $33.7 \%$ in JX-1 cells, but less than 5\% apoptotic cells were found in JX-0 and BEL-7404 cells. Data obtained with DNA fragmentation Elisa test also supported that JX-1 cells had a higher rate of apoptotic cell death than the control vector transfectants, especially during serum-starvation (Tab 2). Ultrastructure analysis of JX-1 cells demonstrated the occurrence of some characteristic morphologic changes of apoptosis, including condensation and fragmentation of chromatin and formation of apoptotic bodies (Fig 2).

Effects of EGFR on apoptosis is steadily gaining attention in recent years[1013]. EGF can maintain the survival of the mouse embryo cell under serum-free condition, while deprivation of EGF induces these cells undergoing apoptosis[10]. An anti-EGFR monoclonal antibody has been reported to block activation of EGFR 
Fu T et al.

Fig 1. Flow cytometric DNA content analysis of BEL-7404, JX-0 and JX-1 cells. These cells were grown in the absence of serum for $72 \mathrm{~h}$ before harvesting. $\mathrm{X}$ axis represents fluorescence intensity and Y-axis represents cell members.

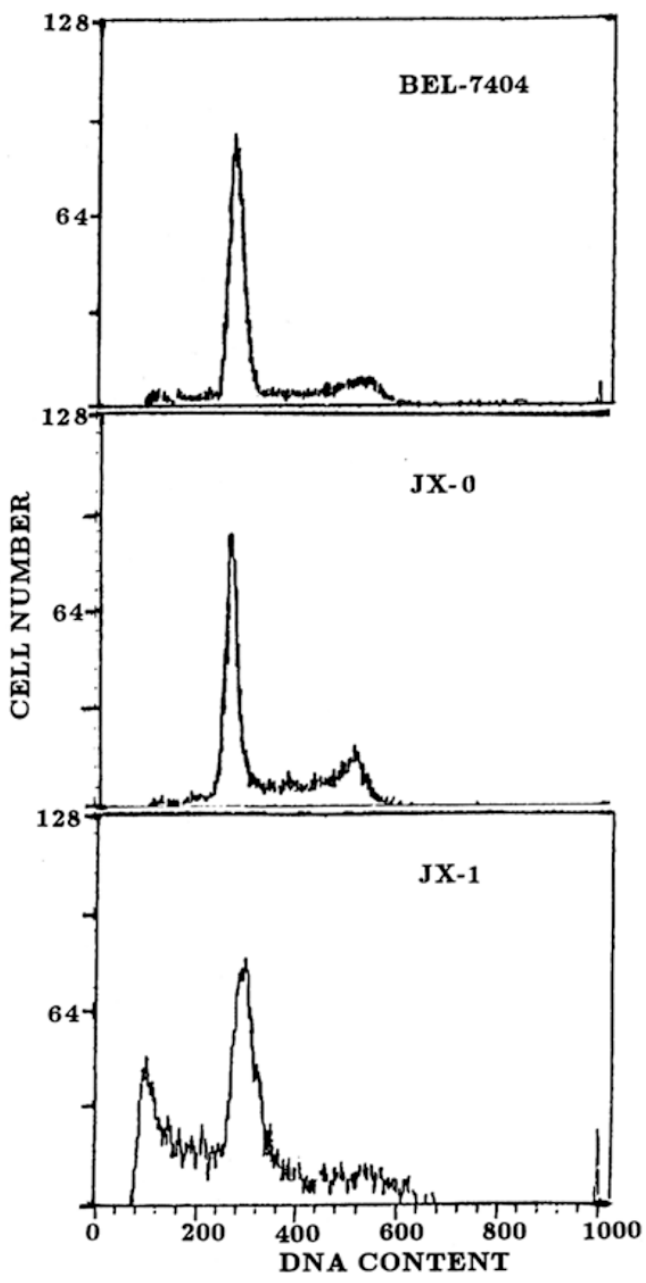

Tab 2. Effects of serum-deprivation on DNA fragmentation in JX-0 and JX-1 cells*

\begin{tabular}{ccc}
\hline Serum-free & DNA Fragmentation (OD, M \pm S.D., $\mathrm{n}=4)$ \\
\cline { 2 - 3 } duration $(\mathrm{h})$ & JX-0 & JX-1 \\
\hline 0 & $0.163 \pm 0.047$ & $0.456 \pm 0.172$ \\
24 & $0.305 \pm 0.062$ & $0.956 \pm 0.117$ \\
48 & $0.278 \pm 0.064$ & $1.134 \pm 0.207$ \\
\hline
\end{tabular}

DNA fragmentation was measured by using the DNA fragmentation Elisa Kit.

tyrosine kinase and induce apoptosis in some human colorectai carcinoma cells[11]. We also observed that the monoclonal antibody against EGFR obviously inhibited the growth and induced the apoptosis of BEL-7404 cells in vitro (unpublished data). However, Armstrong et al. have found that activation of EGFR induces apoptosis in some human breast cancer cells[13]. These discrepancy implies that the 
Antisense EGFR enhances apoptosis in hepatoma cells

EGFR-mediated multiple signal transduction pathways may have different effects on apoptosis.

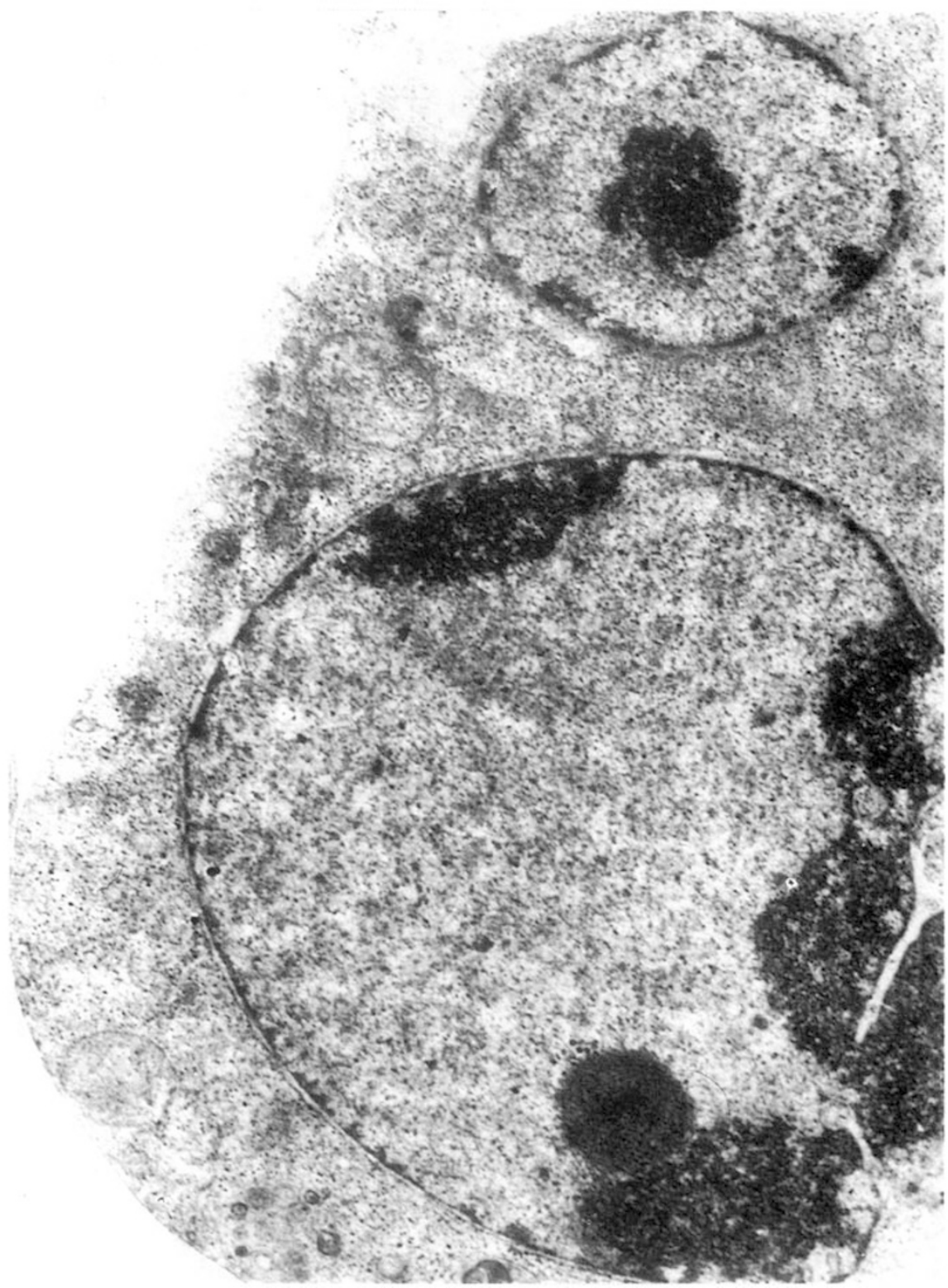

Fig 2. Ultrastructure of JX-1 cells in the absence of serum for $48 \mathrm{~h}$, observed by transmission electron microscope $(\mathrm{x} 6,000)$ 
How does EGFR regulate the growth and apoptosis in human hepatoma cells? EGF binds its receptor and stimulates a series of rapid response including phosphosylation of tyrosine residues within the EGFR itself and many other cellular proteins, hydrolysis of phosphatidyl inosital, release of $\mathrm{Ca}^{2+}$ from intracellular stores and morphological changes. EGFR plays a key role mediating the signal transduction. The deregulation of EGFR expression and function is supposed to disturb the signal pathways in the cells.

As a member of second messagers, calcium may be a sensitive target responsing to the modulation of EGFR activities. We have found that TG, an inhibitor of $\mathrm{Ca}^{2+}$ - ATPase on surface of endoplasmic reticulum induces a transient increase of intracellular $\mathrm{Ca}^{2+}$ in BEL-7404 cells and disturbs the response of intracellular

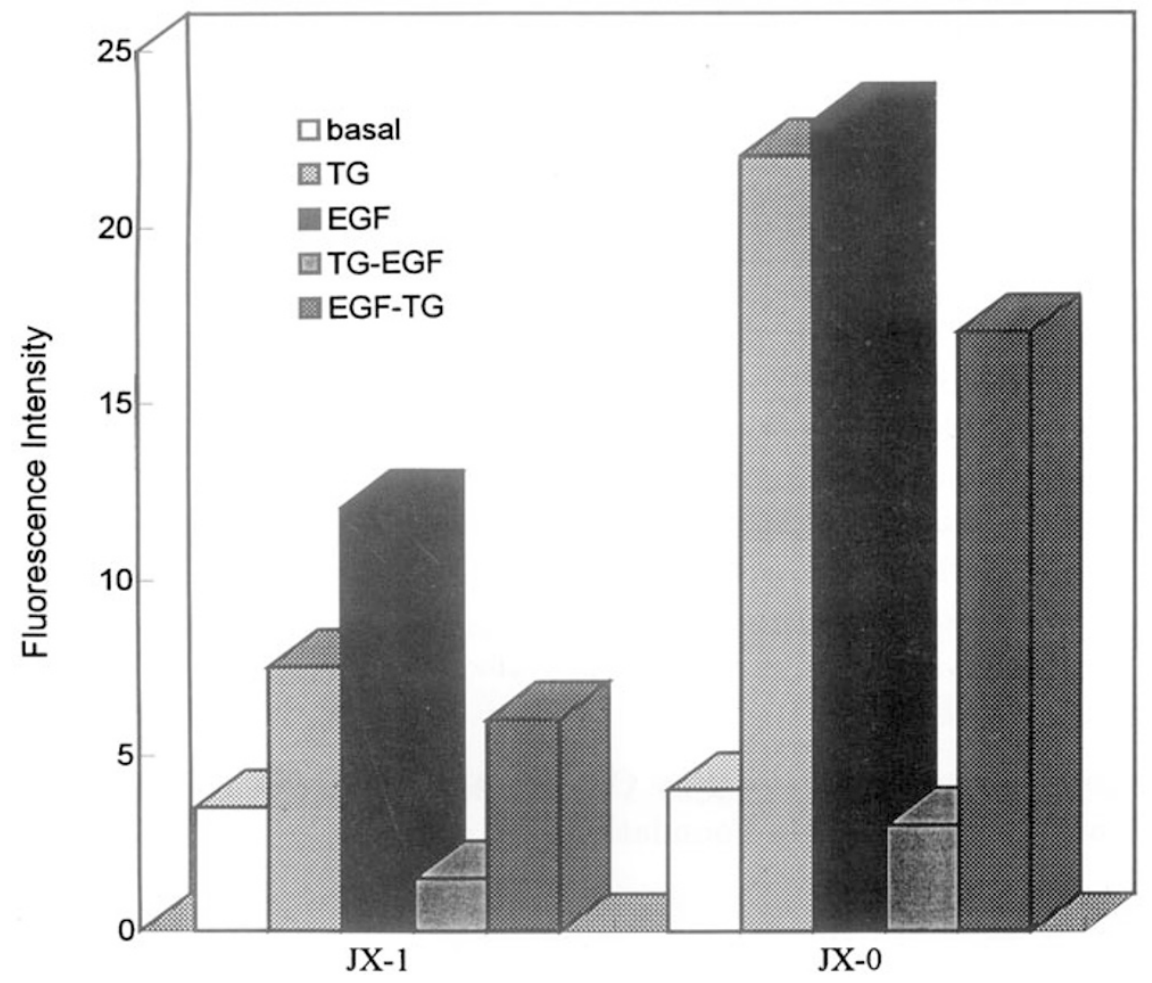

Fig 3. Effects of TG and EGF on intracellular $\mathrm{Ca}^{2+}$ level in JX-0 and JX-1 cells. Intracellular free $\mathrm{Ca}^{2+}$ levels were measured in fluo-3-1oaded cell populations as described in ref. 14. The peaks were recorded after the application of either EGF $(300 \mathrm{ng} / \mathrm{ml})$ or TG $(100 \mathrm{nM})$ for $1 \mathrm{~min}$. EGF-TG: after the pre-incubation with EGF (300 ng/ml) for $10 \mathrm{~min}$, without washing, TG $(100 \mathrm{nM})$ was added for 1 min before intracellular $\mathrm{Ca}^{2+}$ measurement. TG-EGF: after the pre-incubation with TG (100 $\mathrm{n} M$ ) for $20 \mathrm{~min}$, without washing, $\mathrm{EGF}(300 \mathrm{ng} / \mathrm{ml})$ was added for 1 min before intracellular $\mathrm{Ca}^{2+}$ measurement. 
Antisense EGFR enhances apoptosis in hepatoma cells

$\mathrm{Ca}^{2+}$ following the stimulation with EGF, due to the depletion of the intracellular $\mathrm{Ca}^{2+}$ storage in endoplasmic reticulum[14, 15]. In this study, we compared the intracellular $\mathrm{Ca}^{2+}$ level and response to the induction of EGF and TG treatments in antisense EGFR transfected JX-1 cells and control vector transfected JX-0 cells. As shown in Fig 3, short-term treatment of $100 \mathrm{n} M$ TG or $300 \mathrm{ng} / \mathrm{ml}$ EGF induced the release of $\mathrm{Ca}^{2+}$ in both JX-1 and JX-0 cells. The response was much weaker in JX-1 cells than in JX-0 cells by measuring fluorescence intensity. After EGF pre-treatment, JX-1 and JX-0 cells were still sensitive to TG induced stimulation followed by the release $\mathrm{Ca}^{2+}$ with a similar pattern as to TG treatment alone, but a much higher level of intracellular $\mathrm{Ca}^{2+}$ intensity was found in JX-0 cells than in JX-1 cells. However, TG pre-treatment obviously depleted the intracellular $\mathrm{Ca}^{2+}$ storage in the endoplasmic reticulum of the cells resulting in the loss of responsiveness to EGF induction in both JX-1 and JX-0 cells. These results suggested that the antisense EGFR vector transfected JX-1 cells might possess an decreased intracellular $\mathrm{Ca}^{2+}$ pool content in endoplasmic reticulum of the cells under the control of an unknown cascade reactions originating from the interrupted EGFR expression and function triggered by antisense EGFR sequence. It has been shown that the sustained elevation of cytosolic $\mathrm{Ca}^{2+}$ is capable of inducing apoptosis[16]. On the other hand, alteration of intracellular $\mathrm{Ca}^{2+}$ pool content has been considered to be linked to the control of cell growth[17] as well as apoptosis[18, 19]. Our results in this report would support the notion that the alteration of intracellular $\mathrm{Ca}^{2+}$ pool content mediated by antisense EGFR sequence may interfere the signal pathways in regulating and enhancing the process of apoptosis in the JX-l cells. Therefore, the link between EGF/EGFR signaling and intracellular $\mathrm{Ca}^{2+}$ level in the induction of apoptosis of JX-1 cells as well as the exact role played by antisense EGFR remain an attractive target for the future studies.

\section{ACKNOWLEGEMENTS}

The project is supported in part by Chinese Academy of Science and Shanghai Joint Laboratory of Life Science Foundation.

\section{REFERENCES}

[1] Williams GT, Smith CA. Molecular regulation of apoptosis: Genetic control on cell death. Cell 1993; 74:777-9.

[2] Barinaga M. Cell suicide: By ICE, not fire. Science 1994; 263:754-6.

[3] Ellis RE, Yuan J, Horvitz Hr. Mechanisms and functions of cell death. Annu Rev Cell Biol 1991; 7:663-98.

[4] Thompson CB. Apoptosis in the pathogenesis and treatment of disease. Science 1995; 267:145662.

[5] Grullick WJ. Prevalence of aberrant expression of the epidermal growth factor receptor in human cancers. Br Med Bull 1991; 47:87-98.

[6] Downward J, Yarden Y, Mayes E, Scrace G, Totty N, Stockwell P, et al. Close similarity of 
Fu T et al.

epidermal growth factor receptor and v-erbB oncogene protein sequences. Nature 1984; 307:5217.

[7] Mendelsohn J. The epidermal growth factor receptor as a target for therapy with antireceptor monoclonal antibodies. Semin. Cancer Biol 1990; 1:339-44.

[8] Xu Y, Jiang W, Peng S. EGFR expression and EGF stimulation of proliferation in human liver carcinoma cells. Acta Biol Exp Sin 1989; 22:445-53.

[9] Xu Y, Jiang W, Peng S, Chen Y. Antisense EGFR sequence reverses the growth properties of human liver carcinoma cell line BEL-7404 in vitro. Cell Res 1993; 3:75-83.

[10] Rawson CL, Loo DT, duimstra JD, Hedsrom OR, Schmid ES, Barnes DW. Death of serum-free mouse embryo cells caused by epidermal growth factor deprivation. J Cell Biol 1991; 113:671-80.

[11] Wu X, Fan Z, Masui H, Rosen N, Medelsohn J. Apoptosis induced by an anti-epidermal growth receptor monoclonal antibody in a human colorectal carcinoma cell line and its delay by insulin. J Clin Invest 1995; 95:1897-1905.

[12] Fath I, Schweighoffer F, Rey I, Multon MC, Boiziau J, Duchesne M, Tocque B. Cloning of a Grb2 isoform with apoptotic properties. Science 1994; 264:971-4.

[13] Armstrong DK, Kaufmann SH, Ottaviano YL, Furuya Y, Buckley JA, Isaacs JT, Davidson NE. Epidermal growth factor-mediated apoptosis of MDA-MB-468 human breast cancer cells. Cancer Res 1994; 54:5280-3.

[14] Fu T, Xu Y, Jiang W, Zhang H, Zhu P, Wu J. EGF receptor-mediated intracellular calcium increase in human hepatoma BEL-7404 cells. Cell Res 1994; 4:145-53.

[15] Gu J, Liu H, Fu T. Xu Y. Thapsigargin increases apoptotic cell death in human hepatoma BEL-7404 cells. Cell Res 1995; 5:59-65.

[16] Lee S, Chris takos S, Small MB. Apoptosis and signal transduction: clues to molecular mechanism. Current Opinion in Cell Biol 1993; 5:286-91.

[17] Short AD, Bian J, Ghosh TK, Waldron RT, Rybak SL, Gill DL. Intracellular $\mathrm{Ca}^{2+}$ pool content is linked to control of cell growth. Proc Natl Acad Sci USA 1993; 90:4986-90.

[18] Lam M, Dubyak G, Chen L, Nunez G, Miesfeld R, Distelhorst CW. Evidence that Bcl-2 represses apoptosis by regulating endoplasmic reticulum-associated $\mathrm{Ca}^{2+}$ fluxes. Proc Natl Acad Sci USA 1994; 91:6569-73.

[19] Kaneho Y, Tsukamoto A. Thapsigargin-induced persistent intracellular calcium pool depletion and apoptosis in human hepatoma cells. Cancer Lett 1994; 79:147-55.

Received 25-10-1996. Revised 27-11-1996. Accepted 30-11-1996. 